\title{
O processo de enfermagem na concepção de profissionais de Enfermagem de um hospital de ensino
}

\author{
The nursing process in the opinion of the nursing staff of a teaching hospital \\ El proceso de enfermería en la opinión del equipo de enfermería de un hospital universitario
}

\author{
Marília Fernandes Gonzaga de Souza', Ana Dulce Batista dos Santos', Akemi Iwata Monteiro"I \\ ' Universidade Federal do Rio Grande do Norte, Hospital de Pediatria Professor Heriberto Ferreira Bezerra. \\ Parnamirim-RN, Brasil. \\ " Universidade Federal do Rio Grande do Norte, Departamento de Enfermagem, \\ Programa de Pós-Graduação em Enfermagem. Natal-RN, Brasil.
}

\author{
Submissão: 07-04-2011 Aprovação: 01-04-2013
}

\section{RESUMO}

Este estudo objetiva descrever as concepções dos profissionais de Enfermagem sobre o processo de enfermagem, a fim de embasar as ações de implementação do processo na instituição do estudo. Trata-se de um estudo qualitativo, desenvolvido durante uma pesquisa ação, com a equipe de Enfermagem de um hospital pediátrico de ensino. O processo de enfermagem é concebido como um instrumento para organizar a assistência e prescrever os cuidados de Enfermagem. As rotinas de trabalho evidenciam as atividades técnicas. As expectativas com a implementação do processo de enfermagem envolvem a melhoria da qualidade da assistência. O estudo reforça a importância de incluir os membros da equipe de enfermagem, na implementação das etapas do processo de enfermagem e aponta o desafio de enfrentar as rotinas tecnicistas.

Descritores: Processos de Enfermagem; Sistema de Assistência de Enfermagem; Enfermagem.

\begin{abstract}
The aim of this study was to describe the nursing professionals' opinion on the nursing process, in order to base the actions of the implementation of the process in our institution. This is a qualitative study, carried out during an action research with the nursing staff of a pediatric teaching hospital. The nursing process is designed as a tool to organize care and prescribe nursing care. Work routines show the technical activities. Expectations with the implementation of nursing process involve improving the quality of care. The study reinforces the importance of including members of the nursing staff in the implementation stage of the nursing process and shows the challenge of facing the tecnicists routines.
\end{abstract}

Key words: Nursing Process; Nursing Assistance System; Nursing.

\section{RESUMEN}

Este estudio objetivó describir las opiniones de los profesionales de enfermería acerca del proceso de enfermería, con el fin de fundamentar las acciones de implementación del proceso en un hospital de enseñanza pediátrica. Se trata de un estudio cualitativo, realizado durante una investigación-acción con el personal de enfermería de un hospital de enseñanza pediátrica. El proceso de enfermería se ha diseñado como una herramienta para organizar la atención y prescribir los cuidados de enfermería. Rutinas de trabajo demuestran las actividades técnicas. Las expectativas con la aplicación del proceso de enfermería envuelven la mejora de la calidad de la atención. El estudio refuerza la importancia de incluir a los miembros del equipo de enfermería, en la ejecución de los pasos del proceso y apunta el reto de enfrentar las rutinas tecnicistas.

Palabras clave: Procesos de Enfermería; Sistema de Asistencia de Enfermería; Enfermería.

Extraído de: Souza MFG. Sistematização da Assistência de Enfermagem (SAE) em um hospital pediátrico de ensino: uma pesquisa-ação. (Dissertação de Mestrado) Programa de Pós-Graduação em Enfermagem, Universidade Federal do Rio Grande do Norte, 2010. Natal-RN, Brasil. 


\section{INTRODUÇÃO}

O cuidado humano tem sido um tema presente nas discussões sobre o processo de trabalho em saúde e, mais particularmente, da Enfermagem. Sabe-se que muitas profissões estão pautadas no cuidado, porém a Enfermagem tem sido mais frequentemente associada a esta temática desde o seu surgimento na sociedade.

Com o desenvolvimento da ciência, muitos conhecimentos foram produzidos pela Enfermagem, como o processo de enfermagem, que pode ser descrito como um instrumento utilizado para as ações do cuidado. É através dele que o enfermeiro percebe os problemas de saúde, planeja, implementa as ações e avalia os resultados. Entretanto, em virtude dos modelos de saúde dominantes, a Enfermagem adequou o processo de trabalho aos procedimentos, técnicas e rotinas institucionalizadas nos serviços, distanciando-se do cuidado, e das mudanças que seriam necessárias no cotidiano da assistência e do cuidado, do ensino e da pesquisa para a valorização e crescimento do PE como instrumento para sua prática ${ }^{(1)}$.

Ao vivenciar a Enfermagem na pediatria, percebe-se que a proximidade do profissional com o usuário é uma consequência do cuidado e acredita-se que o PE possa possibilitar a reaproximação do enfermeiro com o cliente na realização do cuidado, representando uma estratégia para o resgate das idéias propostas pelo modelo nightingeliano no início da organização da Enfermagem como profissão.

Foi a partir de Florence Nightingale, que o cuidado ganhou especificidade na divisão do trabalho, sendo reconhecido como atividade necessária e útil para a sociedade e que, para seu exercício, era preciso formação especial e conhecimentos que fundamentassem o agir profissional(2).

Contudo, as bases da Enfermagem foram estruturadas também sobre as influências do paradigma positivista, o qual promoveu a fragmentação do saber, através das especializações e divisões do conhecimento, proporcionando "grandes avanços" nas ciências da saúde. Tal paradigma vem afetando a sociedade em diversos aspectos, inclusive nos modelos de saúde, que foram elaborados e influenciados por fatores sociais, políticos e econômicos de cada sociedade.

São muitas as dificuldades para a saúde, uma vez que a sociedade atual está fortemente ligada às tecnologias materiais que, por sua vez, são influenciadas pelos meios de comunicação, centrada em valores de consumo e no modelo biomédico. Consequentemente, valores como a solidariedade, direito universal à vida digna e ao cuidado não são prioridades, o que tem dificultado a valorização de práticas como as da Enfermagem ${ }^{(2)}$.

Ao aplicar as novas tecnologias em saúde, a Enfermagem passou a executar, na maioria das vezes, ações e procedimentos que vêm trazendo prejuízos à relação de cuidado e, desta forma, distanciou-se do paciente, através de prática de ações automáticas, sem análise crítica das mesmas ${ }^{(3)}$.

O processo de enfermagem representa uma alternativa de reaproximação do enfermeiro com seu cliente, entendendo-se o mesmo com um instrumento metodológico de trabalho, que possibilita a análise crítica sobre as condições de saúde do cliente e efetiva a atuação dos profissionais de Enfermagem ${ }^{(4)}$.
Em estudo realizado em uma unidade de terapia intensiva de um hospital público de Fortaleza-CE, foi observado que, na prática, a não aplicabilidade do processo de enfermagem, conduz a prevalência de ações embasadas no senso comum e não na cientificidade. Assim, com a falta de planejamento das ações, não há harmonia entre ciência e prática, o que dificulta o crescimento cientifico da Enfermagem ${ }^{(5)}$.

Com isto, percebeu-se a necessidade de repensar as ações desenvolvidas nas práticas hospitalares. "Um grande desafio, portanto, é romper com as dicotomias construídas social e historicamente: curativo e preventivo, hospital e saúde pública, cuidar e gerenciar, ensinar e fazer, pesquisar e ensinar, universidade e serviço, teoria e prática, dentre outras"(1).

Nesse sentido, a Enfermagem é uma profissão realizada por trabalhadores qualificados e especializados para desenvolver ações socialmente necessárias. Contudo, essa profissão enfrenta algumas fragilidades, tais como a autonomia profissional, o reconhecimento da utilidade social deste trabalho profissional e o domínio de um campo específico de conhecimentos ${ }^{(2)}$.

O processo de enfermagem é um método aplicado à prática da profissão. Sendo definido em três dimensões: propósito, organização e propriedade. O propósito é focado na individualidade, onde o enfermeiro interage com o cliente, confirmando suas observações com o mesmo para, juntos, utilizarem o processo. A organização refere-se às fases distintas, independentes e inter-relacionadas entre si. Por fim, as propriedades são descritas como intencional, sistemático, dinâmico, interativo, flexível e baseado em teorias ${ }^{(6)}$.

É notório que as etapas existentes no processo de enfermagem divergem entre autores; contudo, é possível identificar semelhança na estrutura sequencial entre elas, como a coleta de dados, a análise, o julgamento clínico, o planejamento da ação, a intervenção e a avaliação dos resultados.

O reconhecimento da importância do processo de enfermagem para a profissão evidencia-se através das legislações instituídas pelas entidades de classe. Desta forma, conhecer as legislações em saúde e as específicas da profissão é imprescindível para a Enfermagem se fortalecer naquilo que defende ${ }^{(1)}$.

As resoluções emitidas pelas entidades reguladoras da Enfermagem amparam as ações que buscam o processo de enfermagem para as discussões e justificam as tentativas de sua implementação nos serviços.

Uma das primeiras legislações que orienta a prática da Sistematização da Assistência de Enfermagem (SAE) no país é a Resolução 272/2002 ${ }^{(7)}$ do Conselho Federal de Enfermagem - COFEN, que determinou a implementação da SAE em toda instituição de saúde, pública e privada. Outra resolução importante para a Enfermagem é a Resolução COFEN $311 / 2007^{(8)}$, que reformulou o Código de Ética dos Profissionais de Enfermagem e trouxe vários artigos ressaltando as responsabilidades referentes aos registros de Enfermagem, cujas determinações só são possíveis com a prática da sistematização. Mais recentemente, é possível citar a Resolução COFEN $358 / 2009^{(9)}$, que revogou a Resolução nº 272/2002, reforçando a necessidade de implementação da SAE nos serviços de saúde e incluiu a responsabilidade dos técnicos e auxiliares de Enfermagem na realização do processo de enfermagem. 
Diante destas colocações, questiona-se a existência de conhecimento da equipe de Enfermagem a cerca do processo de enfermagem, como se caracteriza a rotina de trabalho em uma instituição que não desenvolve as etapas do processo de enfermagem formalmente e o que esta equipe espera deste método a ser introduzido na prática de Enfermagem.

Nesse sentido, o objetivo deste estudo é descrever as concepções dos profissionais de Enfermagem sobre o processo de enfermagem a fim de embasar as ações de implementação do processo na instituição do estudo.

\section{METODOLOGIA}

Trata-se de um estudo de natureza qualitativa, desenvolvido na fase de diagnóstico situacional da pesquisa ação, fase essa que representa a primeira etapa do desenvolvimento do método ${ }^{(10)}$. Este estudo representa a primeira etapa de uma dissertação de mestrado e corresponde ao levantamento de informações, e identificação dos problemas pelos participantes.

O estudo foi desenvolvido no Hospital de Pediatria Professor Heriberto Ferreira Bezerra (HOSPED) Universidade Federal do Rio Grande do Norte (UFRN), envolvendo a equipe de Enfermagem (enfermeiros, técnicos e auxiliares) dessa instituição. $\mathrm{O}$ universo deste estudo foi composto por 11 enfermeiros e 26 técnicos e auxiliares de Enfermagem. Para a seleção dos participantes foi adotado como critério de inclusão: ser profissional de Enfermagem que atue no setor de enfermaria e como critério de exclusão: profissionais que estivessem de férias ou afastados no período do estudo. Assim, participaram deste estudo 8 enfermeiros e 21 auxiliares e técnicos de Enfermagem.

Dos 29 participantes do estudo, quanto ao tipo de vínculo com a instituição, 20 são servidores efetivos, e apenas nove têm vínculo terceirizado. As idades variaram entre 25 e 47 anos. O tempo de trabalho na instituição variou entre 1 e 26 anos, com uma predominância dos funcionários que tem entre 1 a 6 anos (15).

Os dados foram coletados no período de novembro a dezembro de 2009, por meio de um questionário contendo perguntas abertas e fechadas, cujas respostas foram registradas de forma escrita pelo pesquisador. A aplicação desse instrumento ocorreu individualmente a cada participante, nas dependências do hospital.

Para a análise e interpretação dos dados optou-se pela análise de conteúdo ${ }^{(11)}$. Para tanto, as informações coletadas foram digitadas e categorizados, o que resultou em três grandes categorias temáticas. A Categoria 1-Conhecimentos do processo de enfermagem foi subdividida em duas subcategorias: assistência e cuidar; organização e sistematização da assistência. A Categoria 2- Rotinas de trabalho foi subdividida em duas subcategorias: atividades técnicas e assistenciais; atividades técnicas e ações de humanização e educação. A Categoria 3- Expectativas com a implementação da SAE foi subdividida em três subcategorias: melhoria da qualidade da assistência; melhoria nos processos de trabalho e de ensino; crescimento da profissão e capacitação.

No primeiro contato com os sujeitos pesquisados foi exposto o objetivo da ação que visa a implantação da SAE no HOSPED.
O projeto foi previamente aprovado pelo Comitê de Ética em Pesquisa da UFRN, com Parecer $n^{\circ}$ 3002009. Todos os participantes assinaram o termo de consentimento livre e esclarecido. Os trechos dos questionários foram identificados com o código das letras E para enfermeiros e T para técnicos, sendo os primeiros sequenciados por números e os seguintes por letras do alfabeto $(E 1, \ldots, T A, \ldots)$, o que visa preservar a identidade dos participantes obedecendo às normativas éticas da Resolução $n^{\circ}$ 196/96 do Conselho Nacional de Saúde.

\section{RESULTADOS E DISCUSSÕES}

Categoria 1 - Conhecimentos do processo de enfermagem

Dentre os 29 entrevistados, 16 referiram ter conhecimentos sobre o processo de enfermagem. Por meio da análise das respostas aos questionários foi possível evidenciar os conhecimentos da equipe sobre o processo de enfermagem. Esses conhecimentos perpassam a assistência de enfermagem, de modo a envolver a organização e sistematização da assistência como evidenciados nas subcategorias que se seguem, e o cuidar enquanto objeto de trabalho da Enfermagem.

\section{Organização e sistematização da assistência}

Percebe-se que, no processo de trabalho do enfermeiro, cotidianamente ocorre um direcionamento das ações para o foco organizacional da instituição, podendo-se inferir a concepção da competência gerencial que compõe o processo de trabalho, com isso o profissional vê no PE as ações de planejar, organizar e direcionar sua prática.

Além dessas funções, para a equipe de Enfermagem o processo de enfermagem possibilita a elaboração de uma prescrição de Enfermagem com cuidados individualizados, além de viabilizar a melhoria nos registros de Enfermagem e a humanização da assistência. Tais constatações são expressas na concepção da maior parte dos profissionais.

Implementação das ações de Enfermagem de forma organizada. (E3)

São etapas a serem desenvolvidas durante o processo de trabalho que servem para facilitar e melhorar a prática e a assistência de Enfermagem. (E8)

Organização dos serviços de Enfermagem de forma sistematizada, com prescrição dos cuidados de Enfermagem. (TU)

Assim, o processo de enfermagem é percebido pela equipe como um instrumento que tem a função de organizar a assistência de Enfermagem, remetendo a uma função técnica gerencial das ações de Enfermagem. Sobre essa ótica, o processo de trabalho do enfermeiro teve um avanço com a utilização dos conhecimentos da área de administração, pois estes influenciaram na sistematização dos cuidados de enfermagem adotada nas instituições hospitalares, contribuindo para a valorização das ações, maior visibilidade e ampliação dos campos de atuação profissional ${ }^{(12)}$. Estes conhecimentos de chefia/gerência estão diretamente ligados à percepção de poder do papel clínico do 
processo, mesmo quando conhecimentos e atitudes de toda a equipe de Enfermagem são favoráveis a ele ${ }^{(13)}$.

As etapas do processo de enfermagem são uma preocupação para sua implementação, uma vez que elas irão modificar o processo de trabalho empregado habitualmente. Isto implicará em inovações, aprendizado e quebra de paradigmas, tanto internamente quanto no que se refere aos outros membros da equipe de saúde.

Tais constatações reforçam os achados de outros estudos, que identificaram o discurso dos enfermeiros como coerentes com os conceitos do processo de enfermagem, apontando as etapas inerentes a sua implementação, destacando a importância do processo no cuidado individualizado e, ainda, revelando a necessidade de aplicação de uma teoria para guiar e fundamentar as ações durante os cuidados profissionais ${ }^{(14)}$.

Outro achado foi o conhecimento de algumas etapas do processo de enfermagem por parte da equipe de técnicos e auxiliares de Enfermagem. Um estudo com auxiliares e técnicos de enfermagem, demonstrou que estes conhecem quase todas as etapas do processo de enfermagem, conhecimento este adquirido durante a prática profissional; assim, sugere-se a necessidade de inclusão desse conteúdo no currículo de formação desses profissionais, pois, no desenvolver do processo de enfermagem, existem atribuições que são inerentes a toda a equipe de Enfermagem ${ }^{(15)}$.

A confirmação deste dado foi um fator surpresa nesta investigação. A concepção dos auxiliares e técnicos de enfermagem com relação ao processo de enfermagem surpreendeu, pelo fato de sua não execução neste serviço e a existência de práticas isoladas, ainda incompletas, em outras instituições da cidade. Além do fato de que, na realidade de formação desses profissionais, estes conhecimentos ainda não estavam incluídos, tendo sido adquiridos no cotidiano profissional. Percebeu-se uma compreensão das etapas do processo, especialmente no que diz respeito à prescrição de enfermagem e aos registros, principais implicações em seu processo de trabalho com a implementação do processo de enfermagem.

\section{Assistência e cuidar}

A concepção dos enfermeiros sobre o processo de enfermagem com enfoque na organização do serviço revelou uma perda do foco de sua atuação profissional, com valorização das ações que respondam as necessidades administrativas da instituição, em detrimento ao cuidado individualizado e humanizado que deveriam ser objeto da ação. Como consequência, o processo de trabalho de enfermagem costuma ser questionado na prática, tendo suas dificuldades apontadas nos discursos dos enfermeiros. O direcionamento ao cuidado enquanto objeto de trabalho da Enfermagem, passou a ser restrito ao vocabulário de poucos profissionais.

No processo de enfermagem o objeto de trabalho é o cui$\operatorname{dar}(E 7)$

Ver o paciente como um todo, fazendo o exame clínico com o olhar da Enfermagem (TD)
O conceito do processo de enfermagem, aparentemente vago, revelou uma compreensão do objeto do trabalho da Enfermagem direcionado ao cuidar, através da utilização dos saberes próprios, construídos com embasamento científico, com aplicações de teorias.

Admite-se que, apesar do crescente uso das teorias de enfermagem, as práticas ainda permanecem fragmentadas, embasadas em sinais e sintomas da doença, e, na solução dos problemas, prevalece a lógica dos serviços, nem sempre se considerando o atendimento das necessidades de quem recebe o cuidado. Essa se constitui uma das dificuldades encontradas no processo de enfermagem permeado por uma prática clínica de cuidados voltados para tarefas, priorizando os serviços e não as necessidades do paciente, resultando num trabalho automatizado e burocrático ${ }^{(5)}$.

Essa realidade encontrada em muitas instituições brasileiras, de pouca aplicabilidade do processo de enfermagem e das teorias de enfermagem, principalmente em instituições públicas, difere da encontrada em um estudo realizado na Espanha, onde o uso do processo de enfermagem é predominante nos serviços públicos. Devido a maiores investimentos governamentais, 98\% dos estabelecimentos de saúde daquele país executam o processo de enfermagem em todas as suas etapas; e $86 \%$ dos serviços usam uma teoria de Enfermagem ${ }^{(16)}$.

Isto pode explicar a razão pela qual a prescrição de cuidados de enfermagem representa uma quebra de paradigma, com mudança de cultura na instituição e rompimento com o modelo biomédico e, que provavelmente, promoverá confrontos com outras categorias de saúde, devido às decisões prescritivas dos cuidados determinados pelos enfermeiros, apresentando-se como um desafio para a consolidação do processo de enfermagem nas instituições.

\section{Categoria 2 - Rotinas de trabalho}

A rotina atual de trabalho desenvolvida pela equipe de enfermagem dentro da instituição revela um processo de trabalho pautado no desenvolvimento de ações de cunho assistencialista e, em sua maioria, direcionado a ações técnicas. Tais constatações podem ser percebidas nas subcategorias: atividades técnicas e assistenciais; atividades técnicas e ações de humanização e educação.

$\mathrm{Na}$ categoria de atividades técnicas e assistenciais foram relacionadas as ações como verificar sinais vitais, ver funções fisiológicas, preparo e administração de medicamentos, soroterapia, punção venosa, alimentação, anotações, recebimento e passagem de plantão, aprazar medicações, fazer escalas e supervisionar a equipe. A segunda subcategoria englobou, além dos citados anteriormente, cuidados relacionados à humanização, tais como ouvir o paciente, conforto e higiene, trabalhar em equipe, envolver a família no cuidado, realizar educação em serviço, participar de treinamentos e reuniões.

\section{Atividades técnicas e assistenciais}

Os profissionais descrevem sua rotina conforme é preconizado para suas funções, com normas institucionais, ações burocráticas e realização de procedimentos, seguindo este mesmo parâmetro. Ocorre assim, na prática uma valorização 
dos profissionais no desempenho de rotinas e técnicas, gerando dificuldades na busca de inovações e caminhos que favoreçam o cuidado ${ }^{(14)}$.

As rotinas técnicas conforme se reproduzem nos serviços estão presentes nas falas da maioria da equipe de Enfermagem.

Receber e passar plantão, realizar procedimentos de Enfermagem, supervisionar a assistência e a equipe. (E1)

Verificar sinais vitais, preparar medicação, soroterapia, punção venosa, higiene corporal, admissão, controle hídrico, oximetria quando necessário, administração de medicação. (TB)

Receber plantão, verificar SSVV, tirar medicação, administrar, puncionar acesso, pesar paciente, aspirar, fazer curativo, ver TA, dieta, balanço hídrico, evoluir paciente e passar plantão. (TG)

Além das funções técnicas e institucionais pertinentes ao discurso de toda a equipe de Enfermagem, os enfermeiros relatam em sua rotina de trabalho a supervisão da equipe e a realização de procedimentos, citando também o planejamento da assistência, embora não sistematizada, mas que demanda conhecimento técnico e científico, experiência e uma avaliação criteriosa das ações.

A adesão aos moldes da rotina de trabalho da instituição é uma tendência do trabalhador ao ser inserido em um processo de trabalho. Dessa forma, ele tende a aceitá-los sem questionamentos, dando continuidade aos modelos encontrados devido à identificação com o sistema, uma vez que a mudança desta rotina poderia gerar atritos ou desconfortos no ambiente de trabalho ${ }^{(17)}$.

Apesar de criticar a organização do processo de trabalho em saúde em que está inserida, a Enfermagem não relata um processo de trabalho diferente do que está institucionalizado nas instituições hospitalares, mas, por vezes, demonstra o inverso, ou seja, conformismo e adaptação ao que lhe é proposto.

Diante das ações cotidianas desenvolvidas pela equipe de Enfermagem da instituição percebeu-se que nenhuma das categorias profissionais fez referências ao trabalho coordenado entre os membros da equipe, revelando o desenvolvimento de um processo de trabalho individualizado, onde cada categoria se fecha nas suas responsabilidades e atribuições, sem interagir entre si no processo de trabalho em saúde.

O problema da ausência do trabalho em equipe, da falta de corporativismo na Enfermagem, resulta em um trabalho fragmentado, onde cada profissional faz a sua parte, não se importando com o todo, observando-se na prática uma valorização dos profissionais no desempenho de rotinas e técnicas, ocorrendo dificuldades em inovações, novos desafios e busca de caminhos que favoreçam o cuidado(14).

\section{Atividades técnicas e ações de humanização e educação}

As atribuições técnicas da Enfermagem, presentes em sua rotina de trabalho, parte do cotidiano dos membros da equipe. Contudo, a existência de ações de humanização e de educação em saúde e conhecimento científico no discurso de alguns profissionais revela que, de forma paulatina, essas ações estão sendo incorporadas ao cotidiano da instituição como práticas intrínsecas ao cuidado qualificado do cliente.

Visitas ao paciente, diagnosticar suas necessidades e traçar a assistência prestada de acordo com sua necessidade. (E4)

Ver a atribuição, tirar as medicações, verificar SSVV, conversar com pacientes, preparar e administrar medicações, evolução de Enfermagem, ajudar no banho se necessário, dieta, maior atenção aos que estão sem acompanhante. (TD)

O processo de enfermagem com enfoque individualizado, direcionando as ações para as pessoas e não apenas para a doença, possibilita ao enfermeiro a identificação de diagnósticos e problemas reais e potenciais, favorecendo o tratamento, a diminuição do tempo de internação hospitalar, melhoria da comunicação, prevenção de erros e repetições desnecessárias ${ }^{(14)}$.

Um aspecto importante do processo de enfermagem citado pela equipe de técnicos e auxiliares de Enfermagem refere-se à assistência humanizada, acrescentando-se o diálogo e a escuta às atividades técnicas, o que permite inferir a existência de uma equipe comprometida com a individualização e humanização do cuidado ao cliente.

\section{Categoria 3 - Expectativas com a implementação da SAE}

O desafio de reaproximar a Enfermagem do cuidado ao paciente está carreado de obstáculos de diferentes âmbitos: institucionais, gerenciais, de recursos humanos e materiais; entretanto, um fator importante a considerar é a expectativa da equipe de enfermagem quanto ao processo de enfermagem.

A falta de interesse e motivação de alguns profissionais é vista como uma dificuldade para implementação do processo. Mudanças nos discursos e atitudes só ocorrerão com envolvimento e compromisso, por serem elementos essenciais para a superação das dificuldades encontradas nos serviços ${ }^{(14)}$. As atitudes positivas dos profissionais poderão colaborar para que as mudanças trazidas aos serviços com o processo de enfermagem possam ser instituídas de forma mais fácil(13). Assim, nas subcategorias a seguir são reveladas as expectativas da equipe de Enfermagem sobre o processo de enfermagem e as mudanças possíveis com sua implementação.

\section{Melhoria da qualidade da assistência}

A expectativa da melhoria da qualidade com a implementação do processo de enfermagem ficou evidenciada no relato de toda a equipe, pois se previu benefícios para a assistência e para o cliente assistido.

Aperfeiçoar a qualidade da assistência de enfermagem. (TS)

Melhoria da qualidade da assistência e prevenção de riscos assistenciais. (E5)

Melhorar nos cuidados ao paciente, contato, mais escuta, cuidados com a técnica melhorada. (TL) 
Sabe-se que o processo de enfermagem é apontado por muitos como possibilidade da Enfermagem melhorar seu status profissional, devido à aplicação de conhecimentos científicos. Porém, em muitas situações, ele é incorporado à prática de forma ritualizada e mecânica, sendo compreendido por alguns profissionais como alternativa salvadora do cuidado de enfermagem, sem apontar a necessidade de amplo conhecimento das ações nele desenvolvidas ${ }^{(14)}$. Contudo, com o entendimento de que o processo faz parte da rotina e do cotidiano de trabalho, ele não deve ser transformado em um fazer mecânico que não envolva a reflexão e a crítica sobre suas etapas ${ }^{(13)}$.

Os resultados deste estudo estão em consonância com a literatura pesquisada, visto que os enfermeiros compreendem o processo de enfermagem como melhoria da qualidade dos serviços e da assistência prestada, além da aproximação ao cliente, sendo para isso necessário um embasamento científico para que ele possa ser determinante no crescimento profissional.

\section{Melhorias nos processos de trabalho e de ensino}

A melhoria da qualidade do processo de trabalho é esperada a partir da implementação do processo de enfermagem na instituição, uma vez que a equipe de. Outro item abordado pela equipe refere-se ao ensino, sendo esta questão mencionada, provavelmente, por se tratar de uma instituição dessa natureza, com a constante presença de discentes em formação.

Melhorar... a qualidade dos processos no serviço e o ensino de graduação. (E1)

Diminuição do índice de erros, garantia da execução dos cuidados de Enfermagem, organização da assistência, meIhora nos relatórios de Enfermagem. (TU)

A dicotomia entre ensino e serviço é uma constante nas discussões sobre o processo de enfermagem, sendo um obstáculo a ser superado, conforme esperam os profissionais de enfermagem.

É comum a abordagem mais teórica do processo de enfermagem nas instituições de ensino superior, entretanto a não articulação da teoria com a prática, dificulta a percepção dos alunos quanto a aplicação e viabilidade deste instrumento no cotidiano do processo de cuidar ${ }^{(17)}$.

A descrição de um processo de trabalho mais organizado, com possibilidades de redução de erros, minimizando os riscos ao cliente assistido, revela um desejo de sucesso nas ações de implementação do processo de enfermagem.

Isto foi corroborado por outros estudos, quando os auxiliares e técnicos de Enfermagem consideraram o PE importante e positivo, por ampliar a visão do profissional acerca das condições do paciente, além de refletir na organização e otimização da assistência, facilitar a localização de informações no prontuário sobre a evolução e cuidados a serem prestados, além da prescrição de enfermagem dar visibilidade ao estado do paciente e facilitar o planejamento de cada turno de trabalho ${ }^{(15)}$.

\section{Crescimento da profissão e capacitação}

Uma das dificuldades mais apontadas no discurso dos enfermeiros no cotidiano profissional é a falta de reconhecimento de seu papel, tanto pelos usuários quanto por outros membros da equipe, isso pode ser justificado por ainda persistir uma prática empírica, sem o planejamento das ações e sem a aplicação do conhecimento científico na prática do cuidado de Enfermagem.

Modificação para modernizar a Enfermagem e no crescimento da Enfermagem. (TF)

Aprendizado com as novas rotinas, servindo até para aplicar em outras instituições. (TD)

Os profissionais da equipe esperam que o processo de enfermagem possibilite mudanças relacionadas à melhoria da qualidade da assistência, à possibilidade de capacitação e conhecimentos científicos, crescimento da profissão, além de associá-lo à humanização da atenção de enfermagem.

Admite-se que não há um consenso formal entre os profissionais de enfermagem no que se refere ao processo de enfermagem. Assim, foi percebido em um estudo o conflito vivenciado pelo enfermeiro diante da crença no processo de enfermagem, ora demonstrando sentimentos de insatisfação, raiva e frustração, ora demonstrando orgulho, vislumbrando o reconhecimento profissional, a ocupação de um espaço social da profissão, a conquista da autenticidade e liberdade de ações ${ }^{(5)}$.

No entanto, o conhecimento sobre o processo de enfermagem foi vislumbrado por parte da equipe de enfermagem, que o associou coerentemente a qualidade, a capacitações, treinamentos em serviço e conhecimento científico. Esta coerência com a proposta do processo de enfermagem, com aquisição de novos saberes, percebida principalmente, pela equipe de auxiliares e técnicos revela, uma compreensão correta do processo de enfermagem. Embora a maioria tenha declarado não saber do que se tratava o processo de enfermagem nos primeiros questionamentos, demonstraram um desejo ou, talvez, uma necessidade da categoria em capacitações para aprimoramento dos conhecimentos.

\section{CONSIDERAÇÕES FINAIS}

As concepções da equipe sobre o processo de enfermagem revelaram que, apesar da receptividade dos profissionais para seu desenvolvimento e implementação na instituição, os conhecimentos da equipe ainda são escassos, necessitando de aprofundamento no tema com leituras, pesquisas, oficinas e aproximação com outras experiências bem sucedidas.

Apesar do pouco conhecimento sobre o processo de enfermagem demonstrado pela equipe pesquisada, percebeu-se que a visão dos profissionais de nível médio está ampliada em relação aos papéis da Enfermagem. Isso envolve a incorporação nas suas rotinas de trabalho de ações que perpassem as atividades técnicas, abrangendo a humanização e a educação em serviços, gerando expectativas de crescimento profissional e capacitação da equipe com a implementação do processo de enfermagem. 
Diante dessas concepções, é possível reafirmar a importância de englobar todos os membros da equipe de Enfermagem na implementação das etapas do processo de enfermagem, tendo em vista que a presença dos técnicos e auxiliares de Enfermagem é elementar para reaproximação da Enfermagem com o cuidado, posto que esses são responsáveis por grande parte da execução de suas etapas. Espera-se com este estudo reforçar a importância da articulação de toda a equipe de enfermagem, incluindo os técnicos e auxiliares de enfermagem, como fundamentais na concretização das práticas de Sistematização da Assistência de Enfermagem.

\section{REFERÊECIAS}

1. Pereira MJB, Fortuna CM, Mishima SM, Almeida MCP, Matumotolet S. A enfermagem no Brasil no contexto da força de trabalho em saúde: perfil e legislação. Rev Bras Enferm 2009;62(5):771-7.

2. Pires D. A enfermagem enquanto disciplina, profissão e trabalho. Rev Bras Enferm 2009;62(5):739-44.

3. Waldow VR. O cuidado na saúde: as relações entre o eu, o outro e o cosmos. 2. ed. Petrópolis: Vozes; 2004.

4. Leadebal ODCP, Fontes WD, Silva CC. Ensino do processo de enfermagem: planejamento e inserção em matrizes curriculares. Rev Esc Enferm USP 2010;44(1):190-8.

5. Alves AR, Lopes CHAF, Jorge MSB. Significado do processo de enfermagem para enfermeiros de uma unidade de terapia intensiva: uma abordagem interacionista. Rev Esc Enferm USP 2008;42(4):649-55.

6. Iyer PW, Taptich BJ, Bernocchi-Losey D. Processo e diagnóstico em enfermagem. Porto Alegre: Artes Médicas; 1993.

7. Conselho Federal de Enfermagem (COFEN). Resolução $n^{\circ}$. 272/2002, de 27 de agosto de 2002. Dispõe sobre a sistematização da assistência de enfermagem - SAE- nas instituições de saúde brasileiras. [resolução na internet]. Diário Oficial da União 27 ago 2002. [acesso em 20 mar 2010]. Disponível em: http://www.coren-df.org.br/ portal/index.php?option $=$ com_content $\&$ view $=$ article\&i$\mathrm{d}=98$ : resolucao-cofen- 2722002 \&catid $=36$ : resolucaes\&ltemid $=43$

8. Conselho Federal de Enfermagem (COFEN). Resolução n. ${ }^{\circ}$ 311/2007, de 08 de fevereiro de 2007. Dispõe sobre o Código de Ética dos Profissionais de Enfermagem. [resolução na internet]. Diário Oficial da União 2007. [acesso 20 mar 2010] Disponível em: http://site.portalcofen.gov. br/node/4345

9. Conselho Federal de Enfermagem (COFEN). Resolução n. ${ }^{\circ}$ 358/2009, de 15 de outubro de 2009. Dispõe sobre a Sistematização da Assistência de Enfermagem e a implementação do Processo de enfermagem em ambientes, públicos ou privados, em que ocorre o cuidado profissional de Enfermagem, e dá outras providências. [resolução na internet]. Diário Oficial da União 2009. [acesso em 20 mar 2010]. Diponível em: http://www.portalcofen.gov.br/ sitenovo/node/4384

10. Thiollent M. Metodologia da pesquisa-ação. 16. ed. São Paulo: Cortez; 2008.

11. Bardin L. Análise de conteúdo. Lisboa: Edições 70; 1977.

12. Shimbo AY, Lacerda MR, Labronice LM. Processo de trabalho do enfermeiro em unidade de internação hospitalar: desafios de uma administração contemporânea. Cogitare Enferm 2008;13(2):296-300.

13. Guedes ES, Turrini RNT, Sousa RMC, Baltar VT, Cruz DALM. Atitudes dos profissionais de enfermagem relacionadas ao Processo de enfermagem. Rev Esc Enferm USP 2012;46(n. ${ }^{\circ}$ esp.):130-7.

14. Freitas MC, Queiroz TA, Souza JAV. O processo de enfermagem sob a ótica das enfermeiras de uma maternidade. Rev Bras Enferm 2007;60(2):207-12.

15. Longaray VK, Almeida MA, Cezaro P. Processo de enfermagem: reflexões de auxiliares e técnicos. Texto \& Contexto Enferm 2008;17(1):150-7.

16. Huitzi-Egilegor JX, Elorza-Puyadena MI, Urkia-Etxabe JM, Zubero-Linaza J, Zupiria-Gorostidi X. Uso do processo de enfermagem nos serviços públicos e privados de um distrito de saúde. Rev Latino-Am Enferm [periódico na internet]. 2012 set [acesso em 20 mar 2013];20(5). Disponível em: http://www.scielo.br/pdf/rlae/v20n5/pt_12.pdf

17. Pinho LB, Santos SMA, Kantorski LP. Analise do processo de trabalho da enfermagem na unidade de terapia intensiva. Texto \& Contexto Enferm 2007;16(4):703-711. 